\title{
Brauner Osten?
}

\section{Wie neulich mal wieder die DDR besiegt wurde}

Die in der deutschen Öffentlichkeit lange Zeit als migrationsmafiöses „Dönermord“Phänomen verhandelte Reihe neonazistischer Hinrichtungen von neun Männern nicht-deutscher Herkunft in den Jahren 2000 bis 2006 hat die Medien und zumindest vorübergehend auch die Politik in den vergangenen Monaten intensiv beschäftigt. Ein Großteil der Tat-Sachen ist mittlerweile bekannt, nicht wenige der Hintergründe und Umstände dieser - im Rückblick - neuartigen Eskalationsstufe rechter Gewalt im „vereinigten Deutschland" sind zunächst sogar angemessen skandalisiert worden, und dies zumindest bis weit in linksliberale politische Kreise hinein: So etwa in dem Sinne, dass die stereotypbehaftete und menschenfeindliche mediale Etikettierung der Mordserie den Blick auf den tiefverankerten deutschen Alltagsrassismus freigibt; dass das systematische Versagen - so nicht sympathisierende Verleugnen - der Verfassungsschutzorgane nicht nur aufgeklärt gehört, sondern die grundsätzliche Frage nach deren Sinn und Zweck, nach ihrem dem Verfassungsauftrag offensichtlich hohnsprechenden Eigenleben aktualisiert; oder dass eine neuerliche Initiative für ein Verbot der NPD sich aufgrund ihrer personellen Teilidentität mit dem Verfassungsschutz bzw. von ihm bezahlten V-Leuten (V wie Verbrüderung) wohl juristisch kaum wird durchsetzen lassen, die notdürftig angelegte bieder-bürgerliche Maske der Partei und ihrer Führung aber erneut gelüftet worden, ihre politisch-praktische Vernetzung mit der faschistischen Organisations-Subkultur in diesem Lande nunmehr tagesschauamtlich ist. So weit, so "gut".

Eine bedeutsame Dimension des „rechten Terrors" und seiner gesellschaftlichen Verarbeitung jedoch hat seit dem Selbstmord der beiden Täter am 4. November 2011 in Eisenach weniger kritische Aufmerksamkeit auf sich gezogen, und dies sind die gewissermaßen "deutschlandpolitischen" Implikationen des mörderischen Geschehens und seiner politisch-medialen Nachwehen. In der politischen Semantik der Vorwendezeit könnte man sagen, dass die Debatte um die Gewalttaten der sich selbst als „Nationalsozialistischer Untergrund“ (NSU) bezeichnenden „Zwickauer Zelle" ein grelles Schlaglicht auf den Stand der innerdeutschen Beziehungen, ihre Struktur und Dynamik, wirft. In ihrer hochgradigen Komplexität und Ambivalenz einem polemisch-spontanen „Einspruch“ selbstverständlich nicht zugänglich, vermag eine genauere Beobachtung der Mechanismen, denen die diskurspolitische Bearbeitung des vermutlich beinahe gesellschaftsweiten Entsetzens über die herrenmenschlichen Exekutions- 
exkursionen zweier ostdeutscher Neonazis gehorcht, doch vielleicht wenigstens ein lehrreiches Schlaglicht auf die deutschdeutsche Gegenwartsbewältigung zu werfen - und damit auf eine Vergangenheit, die (horribile dictu) nicht vergehen will.

Sozialer Brennpunkt des hier interessierenden Geschehens ist nicht Zwickau, wo das untergetauchte Trio BöhnhardtMundlos-Zschäpe seinen letzten, in Flammen aufgegangenen Schattenwohnsitz genommen hatte, sondern Jena, Heimatstadt der drei Hassgetriebenen und Ende der 1990er Jahre Ausgangspunkt sowohl ihres fast vierzehnjährigen Untergrundlebens wie auch des ebenso langen Fahndungsversagens des "Staatsschutzes“. Anders als Zwickau, Chemnitz oder praktisch jedes beliebige andere ostdeutsche Mittelzentrum galt und gilt Jena als Leuchtturm der unmittelbar nach der Wende politisch imaginierten „blühenden Landschaften“ im Beitrittsgebiet. Dass der „braune Terror“ seine organisationspolitischen Wurzeln in Gestalt des "Thüringer Heimatschutzes" gerade in dem ökonomisch prosperierenden Hochtechnologiestandort geschlagen hatte, dass seine menschenverachtende Brutalität ausgerechnet aus dem Schoß einer dem Anschein nach von akademischbürgerlichen Sozialmilieus dominierten Universitätsstadt kroch, musste den interessierten Beobachter hüben wie drüben auf den ersten Blick überraschen - und bot zugleich das ideale Szenario für eilig produzierte Hintergrundberichte zumal der (im doppelten Sinne: vermeintlich) gesamtdeutschen Qualitätsmedien.

Diesbezüglich paradigmatisch war die öffentlich-rechtliche Aufklärungsarbeit des ZDF-Kulturmagazins ,aspekte“, das nach Bekanntwerden der Jena-Connection den deutsch-bengalischen Schriftsteller Steven Uhly zu einem Blitzbesuch in der Stadt an der Saale bewegte, wo dieser dem redaktionellen Auftrag nachgehen sollte, dem bildungsbürgerlichen TV-Publikum hautnah migrantisches Gefühlsleben in der Löwenhöhle des Neonazismus zu vermitteln. ICE-Fahrer, kommst Du nach Jena und nicht (oder nicht erkennbar) aus Deutschland, dann landest Du keineswegs im „Paradies“: Der Off-Text des parainvestigativen Fernsehbeitrags spielte mit dem Namen des provinzschicken Jenaer Fernbahnhofs um hinlänglich plakativ deutlich zu machen, dass selbst die vor wenigen Jahren zur „Stadt der Wissenschaft“ gekürte Wirkungsstätte Schillers und Goethes, Fichtes und Hegels für Ausländer/innen integraler Teil der „ostdeutschen Angstzone" sei. Entsprechend erleichtert zeigte sich der Hauptdarsteller des kleinen Antiwerbefilmchens denn auch ostentativ, als er nach einigen Stunden Ortstermins endlich wieder auf direktem Wege in seine Wahlheimat München ausreisen durfte: „Ich habe einfach zu viel Angst, um mich hier frei zu bewegen", lautete seine zu Protokoll gegebene Quintessenz aus der gebührenfinanzierten Stippvisite in Dunkeldeutschland.

Was daraufhin in Jena, um Jena und um Jena herum geschah, zeugte von einem polittheatralischen Facettenreichtum, der hier nicht einmal andeutungsweise erfasst werden kann: von dem durchaus berechtigten Protest von Aktivist/innen der lokalen antifaschistischen und radikaldemokratischen Szene gegen die Dethematisierung ihres ebenso zähen wie erfolgreichen Engagements gegen Rechts über die zwar verständliche, aber doch auch hinlänglich unsouverän zur Schau gestellte Sorge der örtlichen Politikelite um das Image des Wirtschafts- und Bildungsstandorts bis hin zu der teils nur peinlichen, teils jedoch aggressiv lokalchauvinistischen Empörung jener selbstgerechten Milieus der Jenaer „Bürgerschaft", die ihr Maß 
an praktischer Zivilcourage damit erfüllt sahen, vereint gegen die Verunglimpfung ihrer trauten, heilen Lebenswelt durch die böse, fremde Schmutzpresse zu Felde zu ziehen. All dies soll hier nicht weiter Thema sein - ebenso wenig wie ein ganzer Strauß an politisch-kulturellen Blüten, die der Jenaer Nährboden der "Zwickauer Zelle" in der Folgezeit und bis zuletzt auf regionaler wie nationaler Ebene darüber hinaus trieb: etwa die Eventisierung und Kollektivierung der "Trauer" um die Opfer der Mordserie in Form eines großen Open-Air-Konzerts, für das politischbürokratisch mobilisiert und organisiert wurde wie einst im Mai für zwanglosgezwungene Arbeiteraufmärsche; die plötzliche, vollkommen unglaubwürdige politische Besorgnis und finanzielle Spendierfreudigkeit einer Landesregierung, die ihre institutionell verankerte Freizügigkeit gegenüber rechten Umtrieben im Lande spontan-ostentativ überkompensieren zu müssen und können meinte; die bestenfalls schleppend, am Ende vermutlich im Sande der wundersamen Kommissionsvermehrung verlaufende politische Aufklärung des wahrscheinlich zu gleichen Teilen auf administrativer Unfähigkeit wie auf ideologischer Dienstfertigkeit der Staatsapparate beruhenden Fahndungs-„,GAU “; die totalitarismustheoretisch zwangsläufige und wettbewerbspolitisch offensichtlich geradezu zwanghafte Forderung nach einem das nunmehr allseits gewünschte NPD-Verbotsverfahren flankierenden

1 Heribert Prantl, „Der deutsche GAU. Wie das systemische Versagen des Verfassungsschutzes aufgeklärt werden muss“, Süddeutsche Zeitung vom 12.1.2012; vgl. ders., „Geheimkommission. Innenminister verliert Überblick bei Klärung der Neonazi-Morde“, Süddeutsche Zeitung vom 9.2.2012.
Verbot gleich auch der Linkspartei durch den Generalsekretär der CSU; oder aber auf einem ganz anderen, aber kaum minder beschämenden Blatt der bundesdeutschen „politischen Kultur" stehend - die aufihre ganz eigene Weise unerträgliche, seit den Tagen der selbsterklärt avantgardistischen „Studentenbewegung“ über Jahrzehnte hinweg eingeübte Arroganz des juste milieu der linksalternativen Hauptstadtszene gegenüber den politischen Bewegungen und sonstigen Bestrebungen in der als strukturell zurückgeblieben gezeichneten (einst „westdeutschen“, nunmehr gesamtdeutschen) Provinz. ${ }^{2}$

Gesellschaftspolitisch von besonderer Bedeutung dürfte vielmehr sein, dass sich die deutsche Öffentlichkeit in der Debatte um die Jena Three wieder einmal auf das Schönste in ihren Vorurteilen vom „braunen Osten “ bestätigt fühlen durfte - und mit dem moralischen Sieg der politischen Massenmeinung über die von Nazinestern durchzogene Ostzone zugleich auch, wie dies seit nunmehr zwei Jahrzehnten in schöner Regelmäßigkeit immer wieder aufs Neue inszeniert wird, der ideelle Gesamtschuldner DDR sein Fett wegkriegte. Müßig darauf zu verweisen - denn in diesem politikhistorischen Spiel geht es erkennbar nicht um Fakten -, dass es offenbar die intensive strategische Vernetzung des Jenaer/Zwickauer Terrortrios mit neonazistischen Akteuren und Strukturen auch in den alten Bundesländern war, die ihm die Durchführung der Mordtaten und die Organisation seiner Untergrundexistenz überhaupt ermöglichte. ${ }^{3}$ Eigentlich

2 Vgl. Vid Silber, „In der akademischen Besatzungszone", die tageszeitung vom 8.12.2011.

3 Vgl. Hans Leyendecker und Tanjev Schultz, „Chronik der verpassten Ge- 
unnötig auch ausdrücklich zu betonen, dass es selbstverständlich ein spezifisch ostdeutsches Neonazimilieu gibt, das eine eigentümliche alltagskulturelle Nähe zu kleinbürgerlich-traditionalistischen Sozialmilieus aufweist und sich durch eine besonders ausgeprägte Gewaltbereitschaft auszeichnet - und das es, dies sei hier sicherheitshalber ausdrücklich gesagt, in keiner Weise zu verharmlosen gilt. Aber derartige Erkenntnisse interessieren die angeblich interessierte Öffentlichkeit ja gerade nicht: Was zählt, sind einfache Wahrheiten, und die vermutlich einfachste lautet, dass die neuen Nazis die alte Saat des Arbeiter- und Bauernstaats aufgehen lassen.

Gelegenheiten, die späten und verderblichen Erbschaften des autoritären DDR-Charakters anzuprangern, gab es in den vergangenen Jahren zuhauf, oder besser: politisch-medial genutzte Anlässe, um soziale Probleme der ostdeutschen Teilgesellschaft als Effekte sozialistischer Politik respektive ideologischer Indoktrination $\mathrm{zu}$ rekonstruieren. So wie die unvermeidlichen „Experten“ bei der Aufdeckung von Kindesmisshandlungen in Mecklenburg-Vorpommern unweigerlich - und anders als bei entsprechenden Vorkommnissen zum Beispiel in Bremen - die frühkindliche Töpfchenpflicht in staatssozialistischen Erziehungsanstalten erklärend in Anschlag bringen, so stellt selbstverständlich auch der mörderische Rassenhassfeldzug aus den Tiefen des ostdeutschen Raumes (in einstweiliger Ermangelung einer Klassenhassthese à la Ernst Nolte) eine willkommene Gelegenheit dar, die Täter - und damit auch deren Opfer - als DDR-Spätgeschädigte zu

legenheiten“, Süddeutsche Zeitung vom 4./5.2.2012. beschreiben. Da passt dann für den gesunden Medienmenschenverstand eines zum anderen und irgendwie alles zusammen: freudlose Enge, elternloser Paternalismus, verordneter Antifaschismus, erzwungener Egalitarismus, geduldeter Rassismus - und fertig ist die Gleichung von den intergenerationalen Langfristeffekten des „menschenverachtenden Systems“.

Immer im Dienste der antisozialistischen Volksaufklärung unterwegs sind dabei die mit viel Geld ausgehaltenen „wissenschaftlichen“ Institutionen der offiziellen DDR-Vergangenheitsüberwältigung - allen voran der berüchtigte „Forschungsverbund SED-Staat" an der Freien Universität Berlin, der sich jüngst etwa mit der erfreulich zügig widerlegten (aber wissenschaftspolitisch selbstverständlich folgenlos gebliebenen) Behauptung blamierte, der Westberliner Polizeimord an Benno Ohnesorg gehe eigentlich aufs Konto der Staatssicherheit. Aber warum auch nicht einfach mal versuchen: Die Öffentlichkeit der Berliner Republik traut der „SED-Diktatur" halt alles, jedenfalls alles Schlechte zu, und wenn die Bundesstiftung zur Aufarbeitung derselben nun ihrem volkspädagogischen Auftrag nachkommt und rasch eine öffentlichkeitswirksame Tagung über das „,braune Erbe der Diktatur“ veranstaltet ${ }^{4}$, dann wird schon was dran sein. Oder? Arbeitslosigkeit, Ausländerfeindlichkeit, Abweichendes Verhalten: An allem sind „40 Jahre Sozialismus" und die Diktatur des Proletariats schuld oder irgendwie mitschuldig oder so - zumindest entsprechende Fragen stellen wird man ja

4 Vgl. Jens Bisky, „'Meine Nazis', 'Deine Nazis' - ein müßiges Spiel. Wie braun ist der Osten? Eine Debatte, die nicht vorankommen will“, Süddeutsche Zeitung vom 1.2.2012. 
wohl müssen, meint selbst die selbsterklärt aufklärerische Frankfurter Rundschau: „Kann es sein, dass die Selbstmythisierung des DDR-Staates zum antifaschistischen Vorbild viel tiefer, weitreichender auch zum Selbstbild der DDR-Bürger geworden ist? ... Warum ist es den Jenensern so wichtig, nicht als 'brauner Fleck' auf der Landkarte zu erscheinen? Könnte es nicht auch sein, dass hinter den heftigen Emotionen eine unverdaute Vergangenheit haust?"5

Die gekonnte Paraphrase unvergessener Jenninger-Rhetorik einmal außer Acht gelassen: Mit unverdauten Vergangenheiten kennt man sich in Deutschland ja aus, und darum machte sich die vereinigte Gesellschaft nach 1989/90 auch - nie wieder! mit Macht an die politische Digestion des autoritären Staatssozialismus. Über all den Fragen nach dem roten Erbe und der braunen Erbfolge desselben aber wurden und werden andere wiederum, deren historischsoziologische Relevanz außer Frage steht, erstaunlicherweise nicht gestellt - oder allenfalls, zumindest dem Sinne nach, an ostdeutschen Küchentischen und Biertresen: Kann es sein, dass die Selbstmythisierung der Bundesrepublik zum politischen, ökonomischen, sozialen, kulturellen und was-einem-sonst-noch-so-einfallen-mag Vorbild viel tiefer, weitreichender auch zum negativen Selbstbild der ehemaligen DDR-Bürger geworden ist? Könnte es nicht sein, dass hinter der ostdeutschen Abwehr gegen die Rede von dem „braunen Osten“ und der "Angstzone Ost" eine unverdaute Vergangenheit haust? Ja, das könnte durchaus sein: Eine zwanzigjährige Vereinigungsvergangenheit nämlich von westdeutscher Siegesgewissheit und ostdeutscher Identitätskrise, westdeutschem

5 Dirk Pilz, „Gibt es eine Angstzone Ost?“, Frankfurter Rundschau vom 7.12.2011.
Überlegenheitsgestus und ostdeutschem Unterlegenheitshabitus. Zwei Jahrzehnte westdeutscher Kolonialisierung und Überschichtung des Ostens, ostdeutscher Diskriminierungs- und Abwertungserfahrungen im „vereinigten Deutschland“. Bald eine Generation des arroganten Desinteresses des westdeutschen Durchschnittsbürgers am ostdeutschen Subventionsempfänger und der erzwungenen Mobilität junger Ostdeutscher in Richtung der westdeutschen Futtertröge. Und dass eine solche Perspektivierung ostdeutscher Erfahrungshorizonte der Nachwendezeit in westdeutschen Ohren wiederum nach undankbaren Schmährufen aus dem Jammertal der Alimentierten klingt, ist nur eine weitere Bestätigung der unaufgeklärten und uneingestandenen strukturellen Machtverhältnisse jener gigantischen politisch-sozialen Biographievernichtungsmaschinerie namens „Wiedervereinigung“.

Und so stünde zu vermuten, dass das neudeutsche Fremd- und Selbstetikettierungsspiel auch in Zukunft munter weitergehen wird - dort der handlungsentlastende Verweis auf die „böhsen Onkelz" von der SED, die in den Köpfen und Seelen der Ostdeutschen noch heute ihr Unwesen treiben, hier die von solchen Diagnosen entkoppelte soziale Realität der ostdeutschen Peripherie, Heimat der armen Verwandten der Beitrittsdemokratie. Auf diese gesellschaftliche Spaltung zu verweisen heißt zugleich, Fragen der politischen Deutungshoheit, der kulturellen Hegemonie und der symbolischen Gewalt zu stellen, die im Schatten einer bestenfalls selbstgerechten Verblendung der westdeutschen politischen, wirtschaftlichen und medialen Eliten öffentlich keine Rolle spielen - aber im Gefühlshaushalt der ostdeutschen Gesellschaft umso mehr präsent sind. Wer vom alltäglichen Rechtsextremismus im Osten und von seiner 
Steigerung in der ritualisierten Vernichtungspraxis der Mördernazis Böhnhardt und Mundlos reden will, darf von zwanzig Jahren kollektiv-individueller ostdeutscher "Wendeerfahrung" nicht schweigen. Aber vielleicht regt sich in der Angstzone Ost ja langsam doch auch eine andere, nicht destruktive Form der Selbstermächtigung. Nicht von Ohnmacht oder von der Ansicht, dass "das System“ an allem schuld ist, zeugt jenes neue Selbstbewusstsein junger Ostdeutscher, das manche darin erkennen wollen, dass etwa mit der Chemnitzer Band „Kraftklub“ deren „Verlierergefühl endlich ins Zentrum der eigenen
Selbstbeschreibung" ${ }^{\text {"6 }}$ rückt - sondern, wenn alles gut geht, von der Geburt einer produktiven politischen Bewegung aus dem Geiste eines kollektiven Minderwertigkeitskomplexes. Wie dem auch sei: Aus soziologischer Warte allemal Stoff genug, einen Forschungsverbund zur Aufarbeitung der Vereinigungsfolgen zu fordern. Nach herkömmlichem politisch-medialem Ritual aber vermutlich eher Anlass zu der öffentlich zu verhandelnden Frage, ob „Kraftklub“ nicht verdächtig nach braunem Erbe der Diktatur klingt.

6 Vgl. Jana Hensel, „Ich bin ein Verlierer, Baby!“, der Freitag vom 9.2.2012.

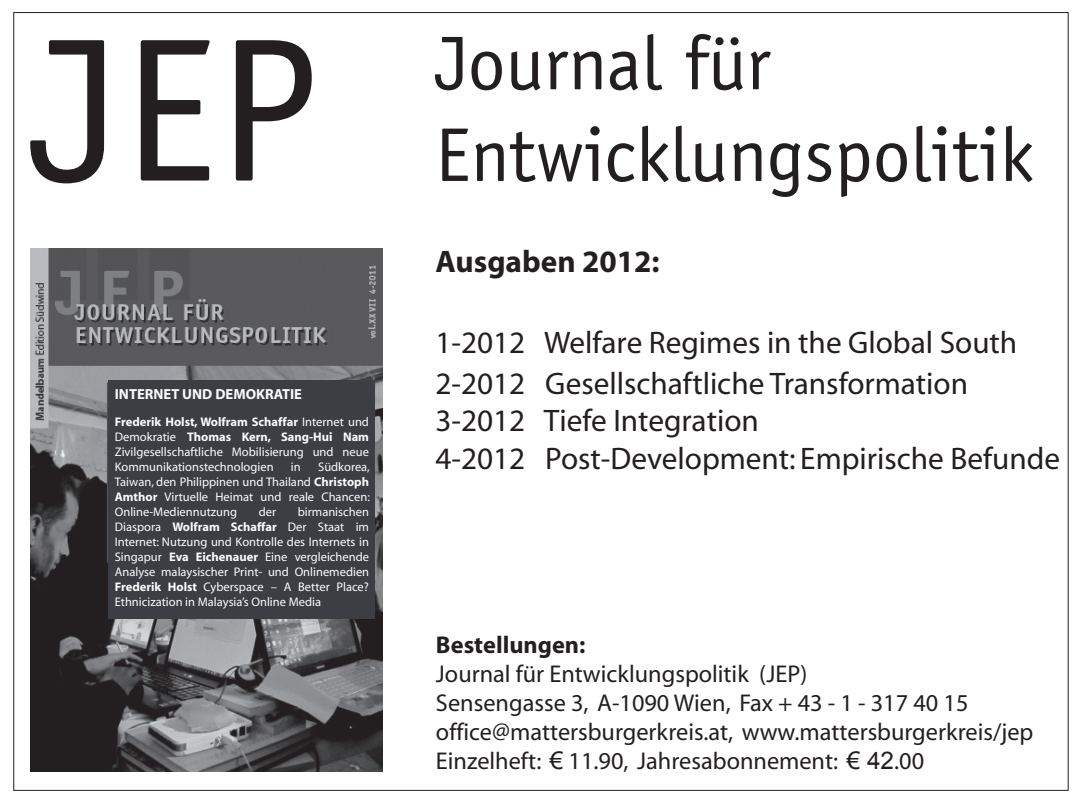

